\title{
Antiglobulins in Nigerians with rheumatoid disease
}

\author{
G. O. OKAFOR*, M. W. TURNER*, AND B. M. GREENWOOD \\ From the Department of Immunology, Institute of Child Health, London* and the Faculty of Medicine, \\ Ahmadu Bello University, Zaria, Nigeria $\dagger$
}

\begin{abstract}
Okafor, G. O., Turner, M. W., and Greenwood, B. M. (1975). Annals of the Rheumatic Diseases, 34, 142. Antiglobulins in Nigerians with rheumatoid disease. The levels of IgG and IgM antiglobulins in the sera of Nigerian patients with seropositive rheumatoid arthritis, seronegative arthritis, and Reiter's syndrome have been studied using an immunosorbent of glutaraldehyde insolubilized human IgG. No conclusion could be reached in the case of IgM antiglobulins because of the relatively high threshold of detectability in the quantitation procedure, but all groups of patients had significantly higher levels of IgG antiglobulins than did a group of healthy Nigerians.
\end{abstract}

Rheumatoid factors (RF) are demonstrable in the sera of $70-80 \%$ of Caucasian patients with rheumatoid arthritis (RA), but the prevalence of RF in Nigerian patients who satisfy the A.R.A criteria for a diagnosis of RA is no greater than in a control group without polyarthritis (Greenwood and Herrick, 1970). In addition, the Nigerian patients show a number of unusual clinical features (Greenwood, 1969a). IgG antiglobulins have been reported in the sera of Caucasian patients with seronegative RA (Torrigiani, Roitt, Lloyd, and Corbett, 1970; Panush, Bianco, and Schur, 1971), so it is possible that such antiglobulins occur in Nigerian patients with RA and may be related to the atypical features of the disease. We have therefore measured IgM and IgG antiglobulins in the sera of Nigerians with RA using an immunosorbent procedure.

\section{Materials and methods}

\section{SERUM SAM PLES}

Serum samples from apparently healthy subjects, from patients with seropositive and seronegative RA, and from patients with Reiter's syndrome were used in the study. The seropositive rheumatoid sera consisted of 5 samples (1 female and 4 males) from patients having an average age of 35 years (range $20-43$ yrs). The seronegative RA sera were obtained from six patients ( 3 females and 3 males) with an average age of 32 years (range 20-44 yrs). The Reiter's group consisted of 4 samples from male patients with an average age of 26 years (range 17-30 yrs). Samples were also obtained from fifteen apparently healthy subjects (8 females and 7 males) having a mean age of 26 years (range 19-35 yrs). The average duration of disease was 16 months (range $5 \mathrm{~m}-4 \mathrm{yrs}$ ) for the seropositive rheumatoid patients, 5 years (range $1 \mathrm{~m}-20$ yrs) for the seronegative rheumatoid patients, and 4 months (range $3 \mathrm{~d}-1$ yr) for the $\vec{\varphi}$ Reiter's syndrome patients. All the RA patients had severe or disease with multiple joint involvement. All patients and controls were resident in or near Zaria, Nigeria.

\section{IMMUNOSORBENT ASSAY}

All sera were investigated by the immunosorbent assay described by Torrigiani and Roitt (1967) as modified by $\varrho$ Panush and others (1971). In this procedure sera are $\overrightarrow{\overrightarrow{0}}$ reacted with insolubilized IgG and bound antibodies are 3 subsequently eluted and measured.

Human IgG (Kabi AB, Stockholm) was insolubilized with glutaraldehyde according to the method of Avrameas and Ternynck (1967) and stored at $4^{\circ} \mathrm{C}$ until required.

Twenty $\mathrm{mg}$ aliquots of insolubilized IgG were incubated with $0.25 \mathrm{ml}$ of serum at $37^{\circ} \mathrm{C}$ for one hour and at $4^{\circ} \mathrm{C} 3$ overnight using a rotary stirrer throughout. The mixture was then centrifuged at $4^{\circ} \mathrm{C}$ for $5 \mathrm{~min}$ at $1000 \mathrm{~g}$ and the supernatant discarded. The immunosorbent was washed with cold saline until the wash fluid gave an optical density of 0.02 or less at $280 \mathrm{~nm}$. Bound immunoglobulin was then $D$ eluted by incubating the immunosorbent with $0.1 \mathrm{~mol} / 1$ 을. glycine-HCl buffer $\mathrm{pH} 2.5$ for $1 \mathrm{~h}$ at $4{ }^{\circ} \mathrm{C}$ with continuous $\overline{\mathrm{N}}$ mixing, followed by centrifugation for $10 \mathrm{~min}$ at $1000 \mathrm{~g}$. क Eluates were neutralized to $\mathrm{pH} 7 \cdot 5$ by the addition of $20 \mu \mathrm{l} \mathrm{O}$ of $1 \mathrm{~mol} / 1 \mathrm{NaOH}$.

The IgG and IgM eluted from the insoluble matrix $\omega_{\sigma}$ were measured by the single radial diffusion technique of Mancini, Carbonara, and Heremans (1965) as modified byㅡㅡ Fahey and McKelvey (1965) using antihuman IgG and $\mathbb{Q}$ IgM from Burroughs Wellcome (Beckenham, England). A standard curve was constructed using 7 dilutions of a serum pool from healthy adults. This standard was cali-우 brated against the International Reference Preparation for human serum immunoglobulins (Rowe, Anderson, $\overrightarrow{\mathbb{D}}$ and Grab, 1970; Rowe, Grab, and Anderson, 1972). 응 In order to facilitate intergroup comparisons, our data are expressed in $\mu \mathrm{g} / \mathrm{ml}$ using approximate conversion $ᄋ$ 
figures obtained by Rowe and others (1972) (1 IU IgG = $80.4 \mu \mathrm{g}$ and $1 \mathrm{IU}$ IgM $=8.4 \mu \mathrm{g}$ ). The lower limits of detectability in the single radial diffusion assay were $15 \mu \mathrm{g} / \mathrm{ml}$ for IgG and $21 \mu \mathrm{g} / \mathrm{ml}$ for IgM. The results were corrected to allow for dilution due to the liquid retained in the washed matrix before addition of the acid buffer. Torrigiani and others (1970) found this dilution factor to be $15 \%$ with BDB insolubilized matrices and we have used a similar correction factor.

The IgG and IgM concentrations in the original serum samples were also measured by single radial diffusion and these values were used to calculate specific activities (see Results).

Statistical analyses were performed where appropriate using the Mann-Whitney $U$ test. $P$ values $<0.05$ were considered significant.

\section{Results}

The results of the estimations of IgG levels in eluates and sera from Nigerian seropositive and seronegative RA patients and Nigerians with Reiter's syndrome, compared with IgG levels for apparently healthy Nigerian subjects, are presented in Fig. 1.

The ranges of the corrected eluate $\mathrm{IgG}$ for the seropositive rheumatoids $(65-158 \mu \mathrm{g} / \mathrm{ml})$, the seronegative rheumatoids $(63-143 \mu \mathrm{g} / \mathrm{ml})$, and patients

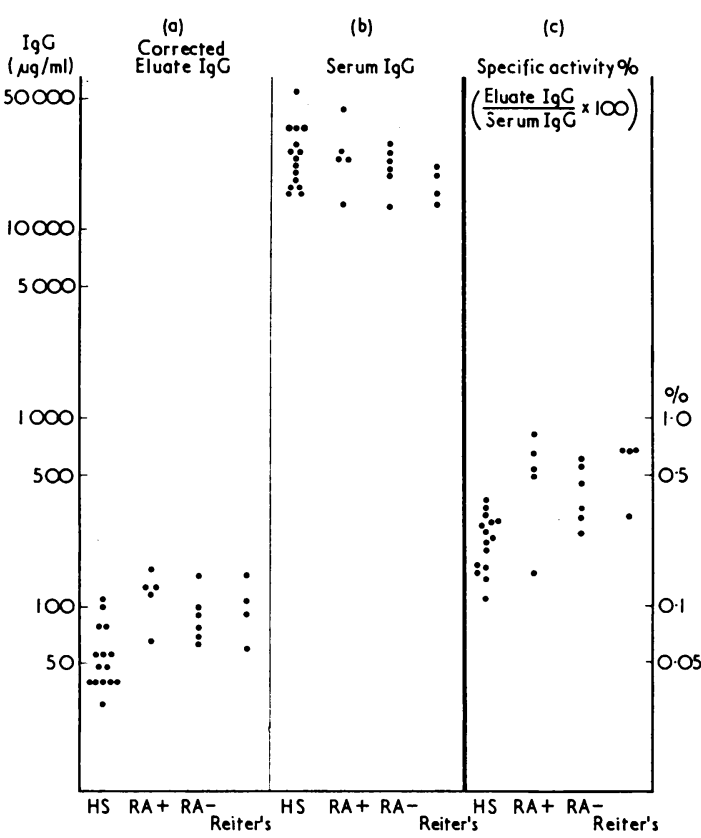

FIG. 1 (a) IgG detected in the eluates from insolubilized human IgG after incubation with sera from apparently healthy adult Nigerians (HS), Nigerian patients with seropositive rheumatoid arthitis $(R A+)$, patients with seronegative rheumatoid arthritis $(R A-)$, and patients with Reiter's disease. Each point represents a determination on a single individual; (b) serum IgG levels in the same individuals; (c) specific activities for each individual with Reiter's syndrome (58-145 $\mu \mathrm{g} / \mathrm{ml})$ were significantly raised compared to the range for healthy subjects $(30-108 \mu \mathrm{g} / \mathrm{ml})(\mathrm{P}<0.001$ for seropositive $\mathrm{RA} ; \mathrm{P}<0.025$ for seronegative RA; and $\mathrm{P}<0.01$ for Reiter's syndrome).

The ranges for serum IgG in the various groups were as follows. Healthy subjects $15000-52000 \mu \mathrm{g} / \mathrm{ml}$; seropositive RA $13000-43000 \mu \mathrm{g} / \mathrm{ml}$; seronegative RA $13400-28600 \mu \mathrm{g} / \mathrm{ml}$; and Reiter's syndrome $13000-21000 \mu \mathrm{g} / \mathrm{ml}$. The range for the group with Reiter's syndrome was significantly lower than the range for healthy subjects $(P<0.05)$, but the other groups were not significantly different.

IgG specific activities, that is the proportion of the total IgG binding to the matrix, were calculated by dividing the IgG concentrations in the eluates by the concentrations in the original sera. As shown in Fig. 1 the ranges of specific activities of all three patient groups were significantly higher than the range of specific activities for healthy subjects ( $P<0.025$ for seropositive RA; $P<0.001$ for seronegative RA; and $P<0.01$ for Reiter's syndrome).

The results of the estimations of IgM levels in eluates and sera from the four groups are shown in

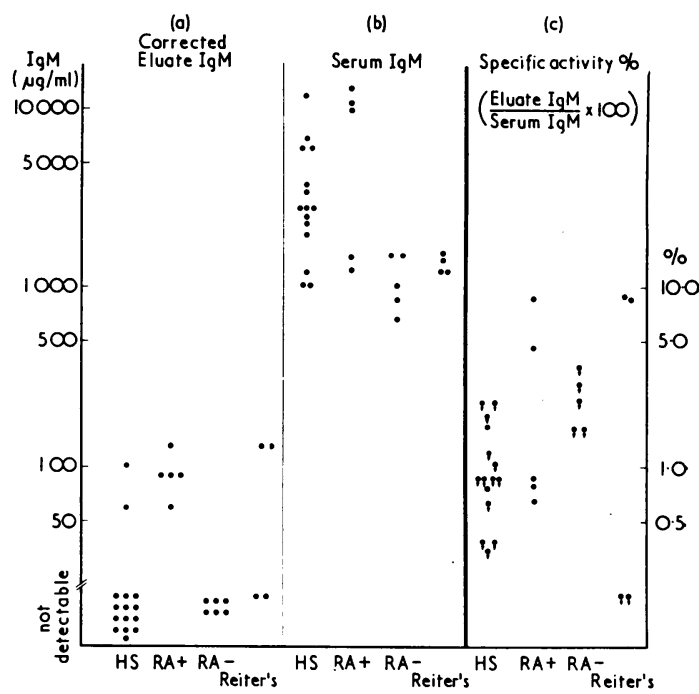

FIG. 2 (a) IgM detected in the eluates for insolubilized human IgG after incubation with sera from apparently healthy adult Nigerians (HS), Nigerian patients with seropositive rheumatoid arthritis $(R A+)$, patients with seronegative rheumatoid arthritis $(R A-)$, and patients with Reiter's disease. Each point represents a determination on a single individual. For statistical comparisons of eluate groups a value of $21 \mu \mathrm{g} / \mathrm{ml}$ (threshold of detectability for the method) was assumed for eluates in which no IgM was detected; (b) serum IgM levels in the same individuals; (there was insufficient sample for assay from one RAindividual); (c) specific activities for each individual. (Statistical comparisons not possible because of large number of indeterminate values) 
Fig. 2. IgM was detected in all eluates from the seropositive rheumatoid patients studied but not in any of the eluates from seronegative patients. High levels of IgM were detected in two patients with Reiter's syndrome but the protein was not found in eluates from sera of two other Reiter's syndrome patients. In eluates from healthy subjects IgM was detected in only two cases. The range of IgM levels in the seropositive group was significantly higher than in the group of healthy subjects $(P<0.01)$.

The ranges for serum IgM in the various groups were as follows. Healthy subjects 1050$12500 \mu \mathrm{g} / \mathrm{ml}$; seropositive RA 1200-12600 $\mu \mathrm{g} / \mathrm{ml}$; seronegative RA $670-1500 \mu \mathrm{g} / \mathrm{ml}$; and Reiter's syndrome $1057-12874 \mu \mathrm{g} / \mathrm{ml}$. The difference between healthy subjects and the seronegative rheumatoid patients and between healthy subjects and Reiter's syndrome patients were both significant by the Mann-Whitney $U$ test $(P<0.01$ and $P<0.05$, respectively).

When specific activities were calculated 2 of the 5 seropositive rheumatoid sera had higher values than any sample from the seronegative rheumatoid or healthy subject groups, but the remainder did not fall above the highest possible value for the sera in which no binding was detected. A statistical comparison was not possible because many of the values for samples from healthy subjects and seronegative RA patients could not be accurately determined.

\section{Discussion}

Rheumatoid factors are frequently found in the serum of Caucasians having no history of rheumatoid disease. In a survey of two British towns, Lawrence, Valkenburg, Tuxford, and Collard (1971) found that only $41 \%$ of individuals with a positive SCAT had RA. Similarly, Greenwood, Muller, and Valkenburg (1971) reported a high prevalence of rheumatoid factor in the sera of apparently healthy Nigerians, and similar findings have been published by other investigators of African communities (Houba and Allison, 1966; Shaper, Kaplan, Mody, and McIntyre, 1968). It is therefore surprising that only $13 \%$ of Nigerian patients with RA gave a positive latex fixation test (Greenwood and Herrick, 1970).

IgG antiglobulins have been reported to be present at increased concentrations in the serum of adult Caucasian patients with seronegative RA (Torrigiani and others, 1970; Panush and others, 1971), in juvenile rheumatoid arthritis (Torrigiani, Ansell, Chown, and Roitt, 1969; Bianco, Panush, Stillman, and Schur, 1971), and in patients with psoriatic arthropathy (Howell, Chamberlain, Perry, Torrigiani, and Roitt, 1972). In all these diseases classical rheumatoid factors are rarely found, and so if antiglobulins are pathogenetic in rheumatic diseases
IgG antiglobulins may provide an alternative mechanism in the seronegative patient.

We have previously studied the specificity of the immunosorbent procedure used in this investigation and shown that much of the difference between rheumatoid and nonrheumatoid sera rests in the concentration of immunoglobulin in the serum (Okafor, Turner, and Soothill, 1975). Specific activity calculations (Eluate Ig/Serum Ig) are needed before evidence of specificity is obtained and when such calculations are applied to the IgG data for Caucasian seropositive rheumatoid patients and healthy subjects the two groups do not differ significantly. In contrast, Nigerian patients with RA (both seropositive and seronegative) and Nigerian patients with Reiter's syndrome have significantly higher levels of IgG antiglobulins than healthy Nigerians.

No conclusion could be reached in the case of IgM specific activities because of the relatively high threshold of detectability in the quantitation procedure. The considerable amount of IgM eluted from the IgG matrix when two sera from healthy Nigerians were studied may be related to the high incidence of rheumatoid factor in healthy Nigerians. It has been suggested by Greenwood and others (1971) that such rheumatoid factor has specificity for homologous IgG whereas rheumatoid factors from Nigerians with RA are able to react with both heterologous and homologous IgG.

There was a nonspecific trend for the patients with seronegative RA and Reiter's syndrome to have lower serum IgG levels than healthy Nigerians. This difference has been shown to be significant in a larger series reported by Greenwood and Herrick (1970) and in patients with actute polyarthritis (Greenwood, 1969b). The significance of these observations is not clear, but one possibility is that the antiglobulins have been particularly effective in immune elimination of antigen, that is host IgG. The low serum IgM in the seronegative rheumatoids is, perhaps, against this idea unless the specificity of the antiglobulin is for antigens shared by IgG and IgM. The precise specificity of the Nigerian IgG antiglobulins needs further investigation using insolubilized immunoglobulin fragments as immunosorbents, as reported elsewhere for Caucasian patients (Okafor and others, 1975). An alternative explanation of the low levels of IgG and IgM in the seronegative Nigerian patients is that it represents an aspect of the immunodeficiency which has been postulated to underly arthritis (Soothill and Steward, 1971).

We thank Professor J. F. Soothill for helpful criticism. G.O. is a Commonwealth Tropical Medicine Research Student supported by the Overseas Development Administration. 


\section{References}

Avrameas, S., AND Ternynck, T. (1967) J. biol. Chem., 242, 1651 (Biologically active water-insoluble protein polymers. I. Their use for isolation of antigens and antibodies)

Bianco, N. E., Panush, R. S., Stillman, J. S., AND Schur, P. H. (1971) Arthr. and Rheum., 14, 685 (Immunologic studies of juvenile rheumatoid arthritis)

FAHEY, J. L., AND MCKelveY, E. M. (1965) J. Immunol., 94, 84 (Quantitative determination of serum immunoglobulins in antibody-agar plates)

GreENwOOd, B. M. (1969a) Ann. rheum. Dis., 28, 488 (Polyarthritis in Western Nigeria. I. Rheumatoid arthritis) (1969b) Quart. J. Med., 38, 295 (Acute tropical polyarthritis) , AND HeRRICK, E. M. (1970) Brit. med.J., 1, 71 (Low incidence of rheumatoid factor and auto antibodies in Nigerian patients with rheumatoid arthritis)

- - Muller, A. S., AND VAlKenburg, H. A. (1971) Clin. exp. Immunol., 9, 161 (Rheumatoid factor in Nigerian sera)

HoubA, V., AND Allison, A. C. (1966) Lancet, 1, 848 (M-antiglobulins (rheumatoid-factor-like globulins) and other gamma globulins in relation to tropical parasitic infections)

Howell, F. A., Chamberlain, M. A., Perry, R. A., Torrigiani, G., and Roitt, I. M. (1972) Ann. rheum. Dis., 31, 129 (IgG antiglobulin levels in patients with psoriatic arthropathy, ankylosing spondylitis, and gout)

Lawrence, J. S., Valkenbrug, H. A., Tuxford, A. F., and Collard, P. J. (1971) Clin. exp. Immunol., 9, 519 (Rheumatoid factor in the United Kingdom. II. Associations with certain infections)

Mancini, G., Carbonara, A. O., AND Heremans, J. F. (1965) Immunochemistry, 2, 235 (Immunochemical quantitation of antigens by single radial immunodiffusion)

OKafor, G., TURner, M. W., AND SoOthILl, J. F. (1975) (In preparation) (Studies on antiglobulins in rheumatoid disease. I. The specificity of glutaraldehyde-insolubilized IgG immunosorbents)

Panush, R. S., Bianco, N. E., AND Schur, P. H. (1971) Arthr. and Rheum., 14, 737 (Serum and synovial fluid IgG, IgA and IgM antigammaglobulins in rheumatoid arthritis)

Rowe, D. S., ANDERSON, S. G., AND GraB, B. (1970) Bull. Wld Hlth Org., 42, 535 (A research standard, for human serum immunoglobulins IgG, IgA and IgM)

- Grab, B., AND ANDERSON, S. G. (1972) Ibid., 46, 67 (An international reference preparation for human serum immunoglobulins $\mathrm{G}, \mathrm{A}$ and $\mathrm{M}$; content of immunoglobulins by weight)

Shaper, A. G., Kaplan, M. H., Mody, N. J., AND MCINTYRe, P. A., (1968) Lancet, 1, 1342 (Malarial antibodies and autoantibodies to heart and other tissues in the immigrant and indigenous peoples of Uganda)

SoOthill, J. F., AND STEWARD, M. W. (1971) Clin. exp. Immunol., 9, 193 (The immunopathological significance of the heterogeneity of antibody affinity)

Torrigiani, G., Ansell, B. M., Chown, E. E. A., ANd Roitt, I. M. (1969) Ann. rheum. Dis., 28, 424 (Raised IgG antiglobulin factors in Still's disease)

_ - AND RoITT, I. M. (1967) Ibid., 26, 334 (Antiglobulin factors in sera from patients with rheumatoid arthritis and normal subjects. Quantitative estimation in different immunoglobulin classes)

- - - Lloyd, K. N., AND CORBETT, M. (1970) Lancet, 1, 14 (Elevated IgG antiglobulins in patients with seronegative rheumatoid arthritis) 